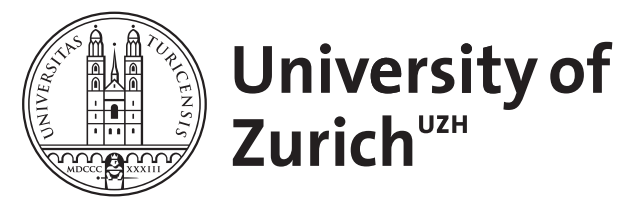

\title{
From lamins to lamina: a structural perspective
}

\author{
Zwerger, Monika ; Medalia, Ohad
}

\begin{abstract}
Lamin proteins are the major constituents of the nuclear lamina, a proteinaceous network that lines the inner nuclear membrane. Primarily, the nuclear lamina provides structural support for the nucleus and the nuclear envelope; however, lamins and their associated proteins are also involved in most of the nuclear processes, including DNA replication and repair, regulation of gene expression, and signaling. Mutations in human lamin A and associated proteins were found to cause a large number of diseases, termed 'laminopathies.' These diseases include muscular dystrophies, lipodystrophies, neuropathies, and premature aging syndromes. Despite the growing number of studies on lamins and their associated proteins, the molecular organization of lamins in health and disease is still elusive. Likewise, there is no comprehensive view how mutations in lamins result in a plethora of diseases, selectively affecting different tissues. Here, we discuss some of the structural aspects of lamins and the nuclear lamina organization, in light of recent results.
\end{abstract}

DOI: https://doi.org/10.1007/s00418-013-1104-y

Posted at the Zurich Open Repository and Archive, University of Zurich

ZORA URL: https://doi.org/10.5167/uzh-79776

Journal Article

Published Version

Originally published at:

Zwerger, Monika; Medalia, Ohad (2013). From lamins to lamina: a structural perspective. Histochemistry and Cell Biology, 140(1):3-12.

DOI: https://doi.org/10.1007/s00418-013-1104-y 


\title{
From lamins to lamina: a structural perspective
}

\author{
Monika Zwerger • Ohad Medalia
}

Accepted: 23 May 2013/Published online: 7 June 2013

(C) Springer-Verlag Berlin Heidelberg 2013

\begin{abstract}
Lamin proteins are the major constituents of the nuclear lamina, a proteinaceous network that lines the inner nuclear membrane. Primarily, the nuclear lamina provides structural support for the nucleus and the nuclear envelope; however, lamins and their associated proteins are also involved in most of the nuclear processes, including DNA replication and repair, regulation of gene expression, and signaling. Mutations in human lamin A and associated proteins were found to cause a large number of diseases, termed 'laminopathies.' These diseases include muscular dystrophies, lipodystrophies, neuropathies, and premature aging syndromes. Despite the growing number of studies on lamins and their associated proteins, the molecular organization of lamins in health and disease is still elusive. Likewise, there is no comprehensive view how mutations in lamins result in a plethora of diseases, selectively affecting different tissues. Here, we discuss some of the structural aspects of lamins and the nuclear lamina organization, in light of recent results.
\end{abstract}

Keywords Nucleus $\cdot$ Nuclear envelope $\cdot$ X-ray crystallography $\cdot \mathrm{In}$ vitro assembly $\cdot$ Xenopus laevis Electron microscopy $\cdot$ Cryo-electron tomograpy

M. Zwerger · O. Medalia $(\bowtie)$

Department of Biochemistry, University of Zurich,

Winterthurerstrasse 190, 8057 Zurich, Switzerland

e-mail: omedalia@bioc.uzh.ch

O. Medalia

Department of Life Sciences and the National Institute for Biotechnology in the Negev, Ben-Gurion University, 84105 Beer-Sheva, Israel

\section{Introduction}

Lamins are type $\mathrm{V}$ intermediate filament (IF) proteins that assemble into a filamentous meshwork underneath the inner nuclear membrane (INM) (Goldman et al. 1986; McKeon et al. 1986). As all IF proteins, lamins share the conserved tripartite structure, consisting of a central $\alpha$ helical coiled-coil domain, flanked by a non-helical $\mathrm{N}$-terminal head and a C-terminal tail domain (Herrmann et al. 2007; Parry 2005). Unlike cytoplasmic IFs, nuclear lamins possess a nuclear localization signal as well as an immunoglobulin (Ig)-fold within their tail domain (DhePaganon et al. 2002; Loewinger and McKeon 1988; Shumaker et al. 2005).

Lamins are classified as A- or B-type lamins. The major isoforms of A-type lamins are lamin A and a shorter isoform lamin $\mathrm{C}$, which both arise from a single gene LMNA, by alternative splicing. The B-type lamins comprise three isoforms of which the most abundant are lamin B1 and lamin B2, encoded by separate genes, $L M N B 1$ and $L M N B 2$, respectively (Lin and Worman 1993; Peter et al. 1989; Vorburger et al. 1989). A and B-type lamins differ in their biochemical properties and expression patterns (Worman et al. 1988). While B-type lamins are expressed in all cells during all stages of development, A-type lamins were not found in embryonic stem cells and are expressed only in later stages of development. Nevertheless, recent studies demonstrated the presence of low levels of lamin $\mathrm{A} / \mathrm{C}$ in mouse embryonic stem cells (Eckersley-Maslin et al. 2013).

Lamin proteins are posttranslationally modified in distinct modification steps. Both A- and B-type lamins contain a carboxy-terminal $\mathrm{CaaX}$ box which undergoes farnesylation of the cysteine, cleavage of the last 3 amino acids (aaX) and methylation of the cysteine. For lamin A but not 
for B-type lamins, an additional proteolytic cleavage step removes the last 15 amino acids, including the farnesyl residue. Therefore, B-type lamins remain permanently farnesylated and associated with the INM (Beck et al. 1990; Kitten and Nigg 1991; Sinensky et al. 1994). Lamin proteins localize mainly at the nuclear periphery, where they are thought to assemble into a protein meshwork underneath the INM. Lamins interact with INM integral proteins as well as with peripheral heterochromatin that is generally gene poor and transcriptionally silent (Guelen et al. 2008; Mendez-Lopez and Worman 2012). A small pool of lamins is also present within the nuclear interior in a more soluble form and seems to serve different functions in a lamina-independent fashion (Dechat et al. 2010b; Shimi et al. 2008).

Mutations in the human LMNA gene cause at least 12 different inherited diseases, collectively termed laminopathies. Laminopathies affect several tissues, for example, muscle, adipose, bone, nerve and skin cells, and fall into one of the four following major disease types: myopathies, including muscular dystrophies and cardiomyopathies, lipodystrophies, neuropathies and premature aging syndromes, such as Hutchison-Gilford progeria syndrome (HGPS) (Rankin and Ellard 2006; Worman 2012). Interestingly, the E145K mutation in LMNA that causes HGPS in humans leads to severely lobulated nuclei, a separation of the A- and B-type lamins, alterations in pericentric heterochromatin, abnormally clustered centromeres and mislocalized telomeres (Taimen et al. 2009). It is still enigmatic how mutations in a single gene can have such variable, tissue-specific effects. Despite the extensive research on lamins, the mechanisms underlying laminopathies are still far from being understood.

Structural analysis of the nuclear lamina is a key element in understanding how mutations may alter the structural organization of the nuclear envelope. Aebi and coworkers visualized the Xenopus laevis oocyte nuclear lamina, by imaging detergent-treated nuclear envelopes using scanning electron microscopy (SEM) (Aebi et al. 1986) (Fig. 1). Although technical limitations prohibit deducing any three-dimensional (3D) structural information, these images represent the overall organization of an amphibian lamin in a very specialized, non-somatic cell. However, the organization of lamin A, lamin C, lamin B1 and lamin B2 in mammalian cells as well as their integration into one meshwork and their connection with INM proteins and peripheral heterochromatin is not known.

Two main factors make it challenging to gain a detailed and realistic map of the mammalian lamina: In vitro investigations of lamin proteins are hindered by their insolubility and their tendency to aggregate into paracrystalline fibers; as all IFs, lamins are strongly resistant to extraction with salt and non-ionic detergent. Therefore,

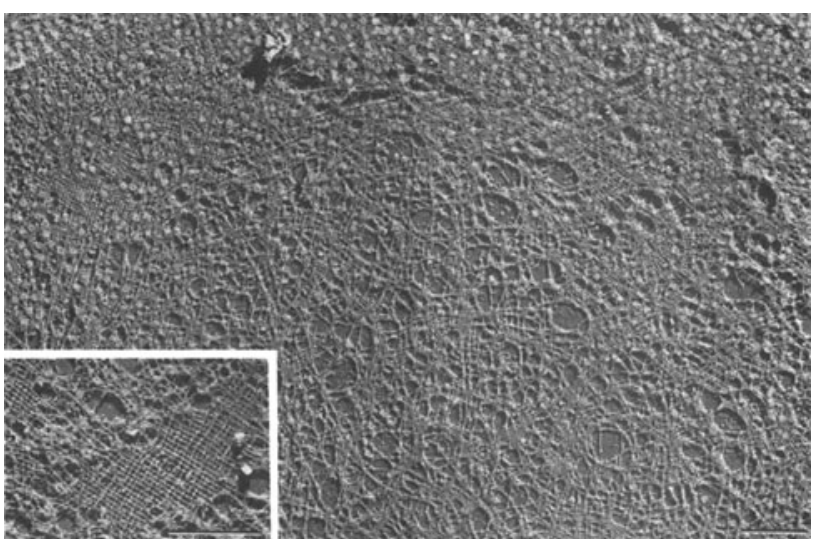

Fig. 1 A view on the nuclear lamina. The nuclear lamina of a freezedried X. laevis oocyte was imaged after membrane extraction. A meshwork of single lamin filaments can be detected, partially covered with arrays of nuclear pore complexes. Inset in an area of the meshwork where nuclear pore complexes have been removed by mechanical forces, the lamina presents as network formed from two sets of parallel filaments organized orthogonally to each other. Scale bar $1 \mu \mathrm{m}$. Reprinted with permission from Macmillan Publishers Ltd: Nature (Aebi et al. 1986), (C)1986

lamins must be generally isolated under denaturing conditions. During refolding in physiological buffers, they rapidly assemble into large molecular structures. In mammalian cells, the lamina is embedded in the dense environment of the nuclear envelope: B-type lamins directly associate with the INM, while all lamins interact with numerous integral INM proteins. Moreover, peripheral heterochromatin closely attaches to the lamina and hinders the visualization of the lamina with high-resolution approaches. Imaging of the lamina hence generally requires isolation either from nuclear membranes or from heterochromatin in order to reveal the lamina structure, and both procedures necessitate harsh treatment of the sample that might cause artifacts. Alternatively, the lamina can be imaged within its native environment, which, however, requires certain markers in order to specifically identify the individual proteins within the crowded nuclear envelope environment. To resolve and interpret the 3D architecture of the nuclear lamina, preexisting information on the supramolecular organization of single lamin proteins is needed.

Here, we focus on several experimental setups currently employed to gain a deeper understanding on lamin assembly and lamina organization. We will discuss the advantages and disadvantages of different approaches and conclude our view on distinct lamin protein assemblies.

\section{Structural determination of lamin proteins}

Structural determination of IF proteins is a challenging task due to the elongated structure of the proteins and their 
tendency to rapidly polymerize. Full length lamins as well as large pieces of the coiled-coil part of the lamin dimer do not arrange into crystals, a prerequisite for determining the atomic structure of proteins by X-ray crystallography, but rather assemble into filamentous or paracrystalline fibers (Herrmann and Aebi 2004). Therefore, a detailed atomic model of lamin proteins is still missing, and a large part of our current knowledge stems from bioinformatic analyses and information on coiled-coils from other proteins (Qin et al. 2011; Strelkov and Burkhard 2002).

Despite the fundamental difficulties to perform X-ray crystallography on lamins, several laboratories applied a 'divide-and-conquer' strategy to reveal their atomic structure, and short fragments of lamins that could be expressed and isolated in a soluble form were analyzed by X-ray crystallography. The first atomic structure of a lamin fragment was obtained from the globular tail domain (amino acids 436-552) of human lamin A/C, showing that this fragment formed a sandwich of two $\beta$-sheets, a structure which is referred to as an Ig domain (Dhe-Paganon et al. 2002). Later, the crystal structure of the lamin B1 Ig domain was determined, and as expected, given the high sequence similarity between the two proteins, the overall structure of the lamin A and B1 globular tail domain is very similar (Ruan et al. 2012). X-ray crystallography was successfully applied to determine the atomic structure of lamin A coil 2B (amino acids 305-387), which represents the last part of the central coiled-coil domain. The analysis of this fragment indicated a left handed, parallel coiled-coil structure (Strelkov et al. 2004). Combined data from this lamin A coil 2B crystal structure and the lamin A coil 1A structure modeled after the crystal structure of another IF family member, vimentin, led to a hypothesis for longitudinal assembly of lamins. According to this hypothesis, the $\mathrm{N}$-terminal part of the coiled-coil rod could overlap and interact with the $\mathrm{C}$-terminal part of a neighboring rod by electrostatic attraction between highly conserved consensus motifs in these regions, thus forming longitudinal head-totail connections of lamin dimers. The crystal structure of a slightly different fragment (amino acids 328-398) that additionally contained a small part of the C-terminal tail domain led to a refinement of this model: Instead of a lefthanded parallel coiled-coil, crystals revealed the presence of two interfaces, a right-handed anti-parallel coiled-coil interface and a left-handed anti-parallel coiled-coil interface (Kapinos et al. 2011). The authors proposed that in addition to the $2-4 \mathrm{~nm}$ overlap between the rods' $\mathrm{N}$ - and C-termini of two dimers (for longitudinal assembly), the C-termini of laterally aligned dimers could partially unzip and engage in additional right-handed coiled-coil interactions between each other, likely in an antiparallel fashion (for lateral assembly) (Heitlinger et al. 1992; Kapinos et al. 2011) (Fig. 2).

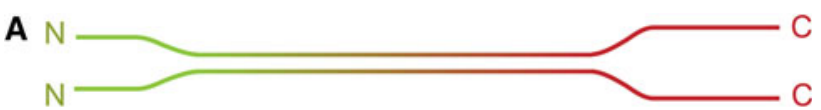

B



Fig. 2 Possible arrangement of coiled-coil domains of lamins to form longitudinal and lateral interactions. a The rod domain of a lamin dimer with 'unzipped' ends; the N-terminal part of the rod colored in green and the C-terminal part in red. $\mathbf{b} \mathrm{X}$-ray crystallography of the C-terminal part of the lamin A coiled-coil rod led to the hypothesis that the ends of the lamin rod can partially 'unzip' and undergo different interactions: (1) a left-handed parallel coiled-coil forms between the rods' $\mathrm{N}$ - and C-termini of two dimers, resulting in an overlap of 2-4 nm of longitudinally assembled dimers. (2) In addition, the C-termini of laterally aligned dimers could engage in additional right-handed coiled-coil interactions between each other, possibly in an antiparallel manner (summarized from Kapinos et al. 2011; Strelkov et al. 2004)

These studies demonstrated that the structural analysis of lamin fragments provides fundamental information on the protein. Finding means to increase the lamin solubility in order to crystallize larger fragments of lamins would be instrumental to yield a better understanding of the lamin structure. Recent studies demonstrate that small molecules can non-covalently bind to proteins or protein fragments that are difficult to crystallize alone, and assist their crystallization (Grubisha et al. 2010; Sennhauser and Grutter 2008). Developing a crystallization 'chaperone' for lamins might thus be a tool toward the structural determination of larger parts of the protein or even full length lamins.

\section{In vitro assembly of lamins}

Mammalian cells can be extracted with buffers containing non-ionic detergents and high concentrations of monovalent ions. By this procedure, most cellular materials are solubilized except for an extraction-resistant fraction of proteins which contains most of the IF proteins. Lamins and all members of the IF family of proteins are therefore regarded as highly insoluble in buffers of physiological ionic strength and $\mathrm{pH}$ (Steinert et al. 1982; Starger et al. 1978).

Purification of lamin proteins, from tissue or from bacteria, generally requires isolation under denaturing conditions. Recombinant lamins expressed in Escherichia coli are deposited in 'inclusion bodies' and are usually isolated by high molar concentrations of chaotropic substances, for example, urea or guanidinium hydrochloride (Herrmann 
et al. 2004). Depending on the reconstitution buffer, lamin proteins will either form soluble dimers, or they further associate to form filaments or paracrystalline fibers, which were suggested to arise from lateral association of filaments (Moir et al. 1990; Karabinos et al. 2003; Aebi et al. 1986).

These physical properties of lamins to assemble into higher-ordered structures were extensively studied. Glycerol-sprayed/rotary metal-shadowed electron microscopy images of lamin dimers revealed the formation of polar dimeric coiled-coil interactions between the two monomers (Aebi et al. 1986; Heitlinger et al. 1991, 1992; Karabinos et al. 2003; Moir et al. 1990) (Fig. 3a). Lamin dimers interact longitudinally by head-to-tail association to form a long polar polymer of dimers that may further assemble laterally into apolar polymers (Ben-Harush et al. 2009) (Fig. 3). This mode of assembly differs from that of cytoplasmic IFs, which initiates by the lateral association of dimers (Herrmann and Aebi 2004; Herrmann et al. 2003).

Transmission electron microscopy (TEM) is instrumental in revealing the fundamental properties and assembly steps of lamins in vitro. Application of classical TEM techniques produces a two-dimensional view on the lamin structure. Recently, 3D views were obtained by applying cryo-electron tomography (cryo-ET) to study filamentous lamin assemblies. Cryo-ET allows revealing the $3 \mathrm{D}$ structure of the cells, organelles and macromolecular assemblies in a native state (Fridman et al. 2012; Yahav et al. 2011). Sample vitrification by rapid freezing ensures close-to-life conditions of biological material, by physical fixation (Dubochet et al. 1988; Yahav et al. 2011). Since neither chemical fixation nor staining is needed, the delicate molecular landscape during sample preparation is preserved under such conditions, allowing for an accurate depiction of macromolecules and lamin structures. CryoET was recently applied to resolve the structure of the Caenorhabditis elegans (Ce-) lamin. These studies revealed that Ce-lamin forms IF-like 10-nm filaments, assembled from three or four tetrameric protofilaments (Fig. 3d) (Ben-Harush et al. 2009).

Although important information can be obtained from in vitro studies, direct conclusions on the in vivo lamin
Fig. 3 In vitro studies of lamins and lamin assemblies. Initial steps of assembly of recombinantly expressed chicken lamin B2 show 'myosin-like' dimers (a arrowheads), short head-totail polymers (a arrows) and longitudinally assembled polar head-to-tail polymers (b). Scale bar $100 \mathrm{~nm}$. (C) Heitlinger et al. (1991). Originally published in J. Cell Biol. 113(3):485-495. Under distinct buffer conditions, the $C$. elegans lamin further assembles to form filamentous structures. c C. elegans lamin filaments negatively stained with uranyl acetate and imaged by TEM. Scale bar $200 \mathrm{~nm}$. c Reprinted from (Karabinos et al. 2003), (2003, with permission from Elsevier. d C. elegans lamin filaments as observed in a 60 -nm-thick section of a cryo-electron tomogram. Filaments show a characteristic, beaded pattern and regular $55 \mathrm{~nm}$ repeats of dimeric subunits (brackets). Scale bar $100 \mathrm{~nm}$. Reprinted from (Ben-Harush et al. 2009), (C) 2009, with permission from Elsevier
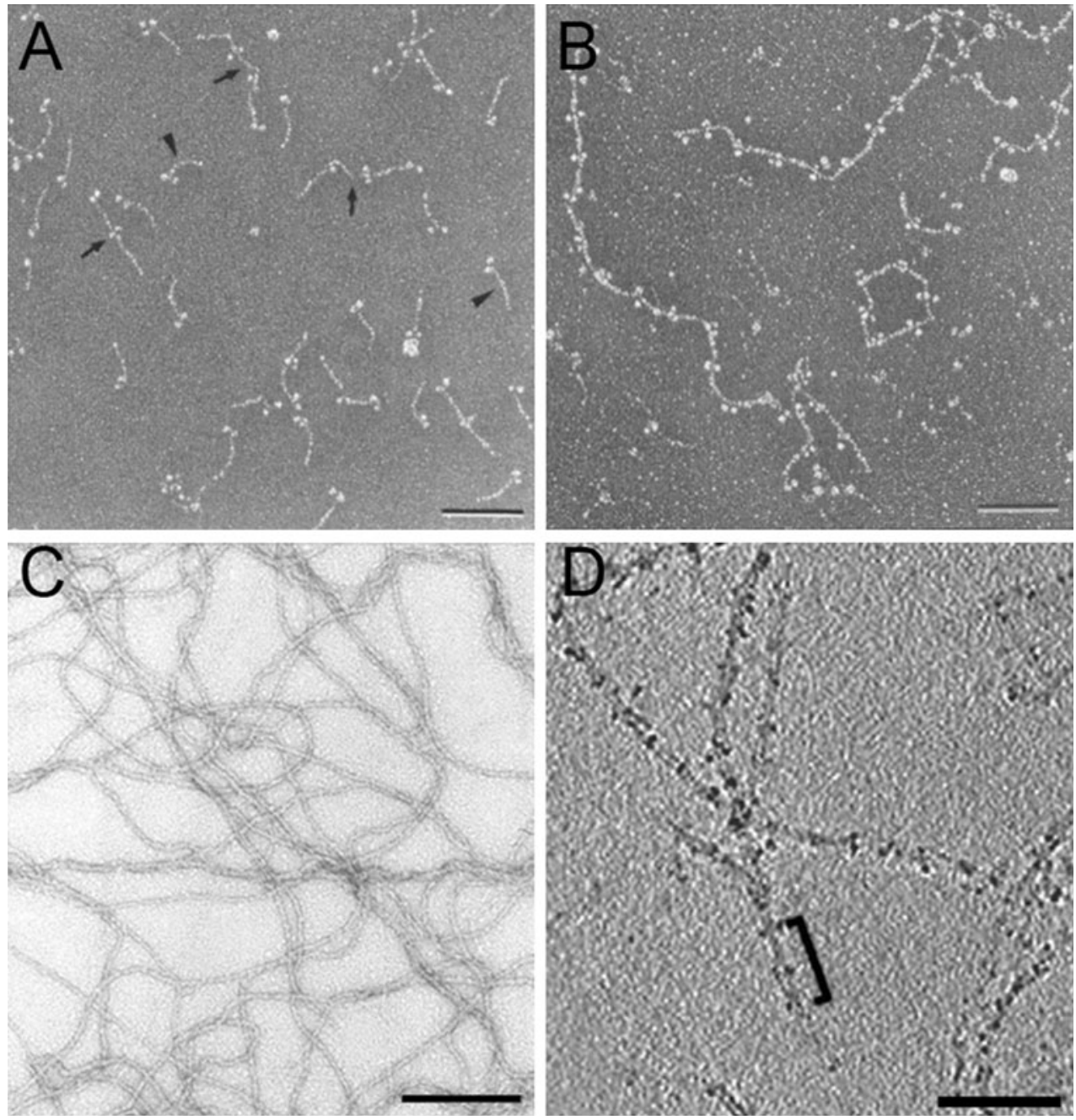
assembly have to be taken with caution. Multiple factors present in a native cellular environment are absent in the test tube. As an example, B-type lamins are attached to nuclear membranes by their farnesyl residue within the cells, and therefore, in vivo, certain interactions might be spatially restricted by the surface of the INM. Soluble and membrane-bound lamin-binding partners, additional scaffolding networks or chaperones might be crucial for an ordered in vivo lamin assembly. As such, it is plausible that lamin-bound chaperones shield-specific domains thus prevent some molecular interactions of unassembled lamins with other factors. Finally, all studies conducted so far used denaturing agents to isolate lamins, and it is not known whether the reconstituted lamin subunits refold to their natively occurring secondary and tertiary structures, especially for recombinant lamins that do not undergo posttranslational modifications. In summary, in vitro investigation of lamin subunits and their assembly is a valuable tool to understand basic mechanisms of filament formation, and it needs to be determined whether these mechanisms also occur in the living cell.

\section{Xenopus laevis oocyte nuclei as platform for lamin assemblies}

Oocytes from the African clawed frog X. laevis provide a very powerful model system for studying the structure and function of lamins (Stick and Goldberg 2010), given their cellular and nuclear size as well as the fact that their chromatin is not attached to the nuclear membranes (Gall et al. 2004).

Xenopus oocyte nuclei express a single lamin, LIII, which is closely attached to the INM and only becomes visible after lipid extraction with detergents. The structure of this endogenous lamin network was characterized in a landmark study, demonstrating that lamin LIII appears to form two sets of parallel filaments organized orthogonally to each other (Aebi et al. 1986) (Fig. 1). Later work suggested the formation of a single set of parallel filaments, interconnected by thinner, regularly spaced lateral interconnections (Goldberg et al. 2008).

In addition to the analysis of the endogenous LIII lamin network, oocytes also represent an excellent in vivo protein expression system: After microinjection of either DNA or mRNA into the nucleus or the cytoplasm, respectively, the cells synthesize the desired proteins usually at high levels.

Moreover, it was shown that somatic lamin proteins of different organisms can be exogenously expressed in Xenopus oocyes and that they form a lamina network on the top of the endogenous lamin LIII-lamina (Grossman et al. 2012; Kaufmann et al. 2011).
Ectopic expression of human lamin A in oocytes showed a dense lamina layer of $\geq 200 \mathrm{~nm}$ in thickness (Fig. 4a). However, after spreading of human lamin A expressing oocyte nuclei, we found nuclear envelopes that closely resembled non-injected control samples (Fig. 4b, unpublished observations), suggesting that the most of the lamin A network detached. In contrast, expression of human lamin B1 in Xenopus oocytes resulted in the formation of filaments, interconnected to form an extensive network on the top of the nuclear envelope (Fig. 4b).

Mature lamin A, in contrast to lamin B1, does not have a farnesyl residue that interacts with the nuclear membrane. It is therefore plausible that mechanical forces and washing steps during sample preparation result in the removal of the lamin A network from the nuclear envelope. Indeed, coexpression of human lamin A together with emerin, a well-established lamin A interacting transmembrane protein, showed substantial structures attached to the nuclear envelope (Fig. 4b). These observations supported the hypothesis that the lamina formed by human lamin A only loosely attaches to the INM and that a lamin-binding protein can 'anchor' lamin A at the nuclear membrane. Surprisingly, the emerin-lamin A network did not display an obvious filamentous architecture as did lamin B1, but rather formed a protein layer.

On the basis of our observations and studies performed by others (Goldberg et al. 2008; Kaufmann et al. 2011), we hypothesize that lamin $\mathrm{A}$ is fundamentally different from lamin B in its general assembly mode. Higher resolved structures are needed to reveal the organization of assembled A-type lamins and the mode of interaction with the nuclear envelope.

Above-mentioned studies were performed using SEM. SEM analyses provide an excellent tool to characterize the spatial arrangement of lamin filaments within the landscape of a nuclear envelope; however, they require fixation, dehydration and a thin metal coating in order to yield a high enough contrast. Alternatively, spreaded nuclear envelopes can be vitrified and directly analyzed by cryoET. We have utilized this system in order to reveal the architecture of the C. elegans lamin and demonstrated that the nuclear lamina formed by Ce-lamin is composed of 5-6 nm filaments (Fig. 4c) that resemble the tetrameric protofilaments that were previously described (Ben-Harush et al. 2009; Grossman et al. 2012). The flexibility of lamin protofilaments became apparent upon analysis of the persistence length. Surprisingly, the organization of the lamin meshwork is anisotropic, because some areas appear denser than others. Despite the irregularity of the meshwork, many of these protofilaments are oriented in parallel to the plane of the NE, whereas only a small portion arranges diagonally or perpendicularly to the NE plane and serves as link between horizontal protofilament layers and the INM. 


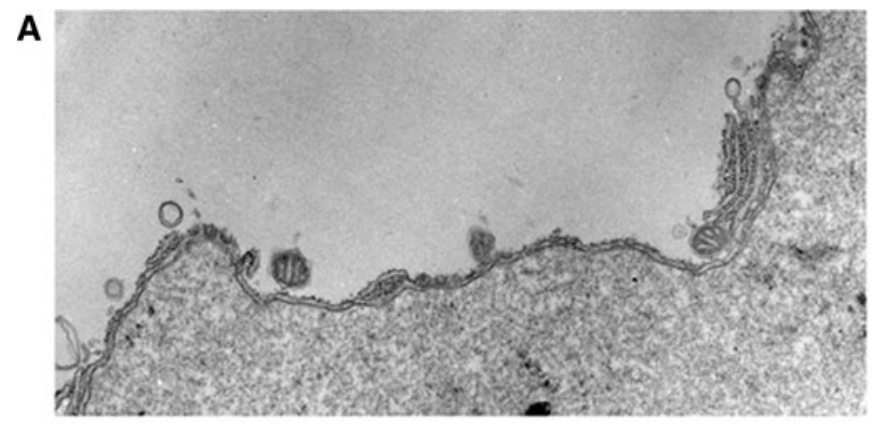

non-injected

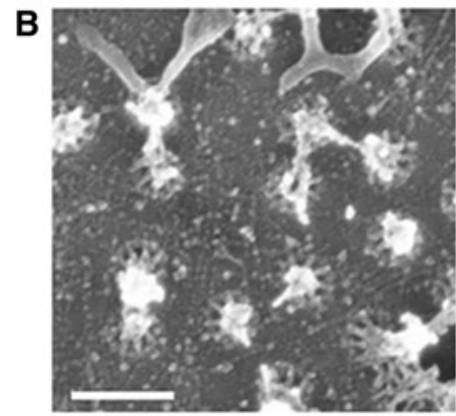

non-injected

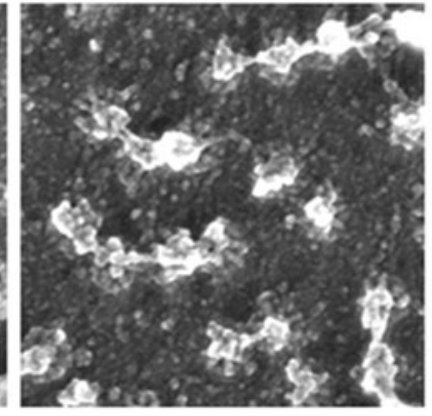

Lamin A

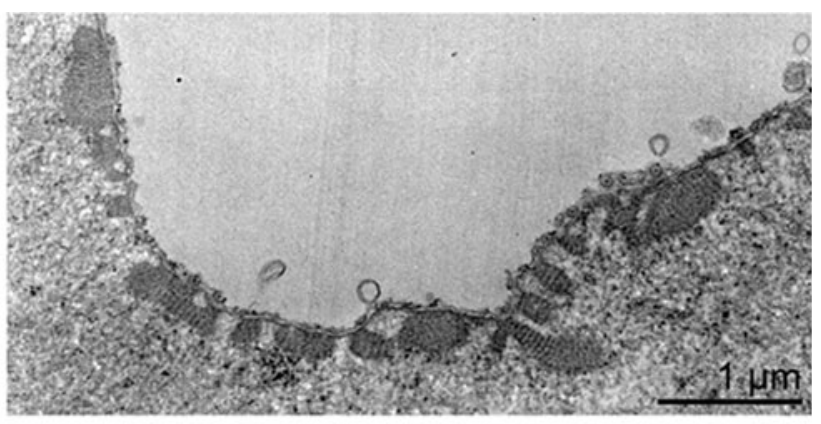

human Lamin A

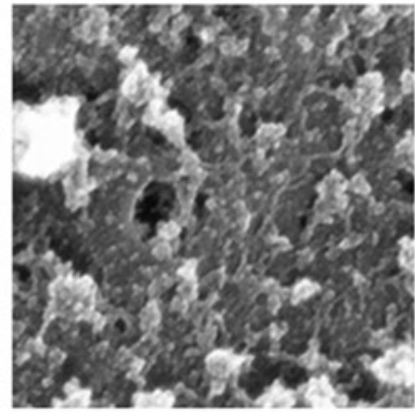

Lamin A + Emerin

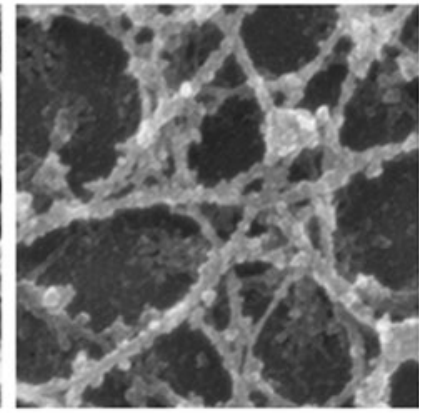

Lamin B1
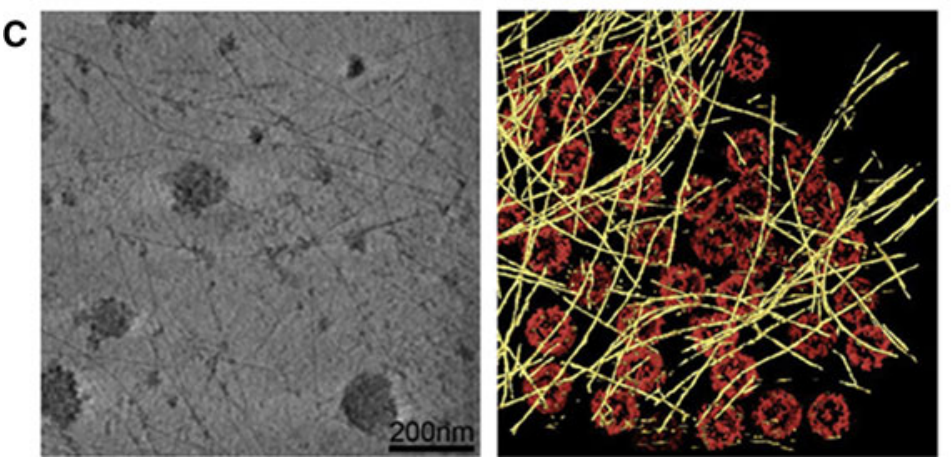

Fig. 4 Studies of lamin assemblies in Xenopus oocytes. a Ultrathin sections of Xenopus oocyte nuclear envelope arrays imaged by TEM. After expression of human lamin A (right image), oocytes exhibit a thick electron-dense layer of lamin A filaments on the top of the endogenous lamina (left image). Scale bar $1 \mu \mathrm{m}$. Reprinted from (Kaufmann et al. 2011). b The nucleoplasmic face of Xenopus nuclear envelope spreads in non-injected oocytes and in oocytes injected with human lamin A, with human lamin A and emerin, or human lamin B1. Spreaded nuclear envelopes were fixed, dehydrated, critical point dried, metal coated and imaged using SEM. Scale bar $200 \mathrm{~nm}$.

The Xenopus model system has provided fundamental insights into the structural organization of lamin networks. Although the nuclear envelope of X. laevis oocytes does not represent the 'native environment,' it still can serve as an 'in vivo' platform, on which laminae from individual lamins as well as from different lamin proteins can be formed. Several different proteins can be coexpressed within a single oocyte nucleus, opening a wide field of possibilities to c Xenopus nuclear envelope spreads after injection of C. elegans lamin analyzed by cryo-ET. The left image represents a $130 \mathrm{~nm}$ tomographic slice through the nuclear side of the nuclear envelope that contains a filamentous meshwork of Ce-lamin filaments. The right image shows a surface-rendered tomogram of the nucleoplasmic side of the nuclear envelope, with Ce-lamin filaments depicted in yellow and NPCs in red. The volume displays $1,310 \times 1,310 \times$ $409 \mathrm{~nm}^{3}$. Reprinted from (Grossman et al. 2012), (C2012, with permission from Elsevier

use this platform for building up lamina networks composed of different proteins: The influence and contribution of different lamins (e.g., human lamin A, lamin B1 and lamin B2), or the influence of INM lamin-binding proteins on the assembled nuclear lamina can be investigated. We therefore believe Xenopus oocytes open yet unexploited possibilities to gain further insight into the organization and crosstalk between lamins and different nuclear envelope components. 


\section{The lamina network in mammalian cells}

The periphery of the nucleus is a tightly packed environment: Numerous integral nuclear envelope proteins, up to several hundreds, are estimated to reside within the INM and many of them interact with the nuclear lamina, chromatin or both (Holmer and Worman 2001; Korfali et al. 2012). The lamina itself is a tight assembly of the lamin proteins lamin A, C, B1 and B2 (Dechat et al. 2010a). Lamins interact, directly and indirectly, with peripheral heterochromatin as well as chromatin-binding and modifying factors (Guelen et al. 2008; Luderus et al. 1992; Mattout et al. 2007). In addition, numerous transcription factors, signaling molecules and nuclear scaffolding components, such as nuclear actin and components of nuclear pore complexes, are associated with lamins (Simon et al. 2010; Lloyd et al. 2002; Kubben et al. 2010).

Visualization of the nuclear lamina from mammalian cells has been conducted by two main methodologies. First, ultrastructural studies can be performed after isolation of the lamina by extracting all attached components. Early studies in this direction included the generation of so-called nuclear ghosts, isolated nuclei that were stripped of DNA and membranes by stepwise treatment with DNAse and detergent and basically represent a 'nuclear pore complexlamina' fraction (Dwyer and Blobel 1976). This technique indeed enabled electron microscopic visualization of the lamina; however, the harsh chemical treatment as well as the removal of connecting or scaffolding components likely causes substantial changes of its native structural organization (Fig. 5a). In a different attempt, the sole removal of membranes from isolated mouse liver nuclei to enable a view on the lamina from the cytoplasmic side did not reveal the filamentous nature of the lamina (Kirschner et al. 1977) (Fig. 5b).

A second approach is the visualization of the lamina network in its native environment in non-extracted cells. For fluorescence microscopy, this requires labeling of lamins with a fluorescent reporter as well as high-resolution microscopy. Conventional confocal laser scanning microscopy (CLSM) performed on nuclei labeled with antibodies against lamin $\mathrm{A} / \mathrm{C}$, lamin $\mathrm{B} 1$ and lamin $\mathrm{B} 2$ revealed individual, discontinuous patches of A- and B-type lamins with occasional points of colocalization. These studies led to the view that A- and B-type lamins form mainly separate but interconnected networks (Shimi et al. 2008). For a more detailed insight into the arrangement of different lamin isoforms, it will be necessary to

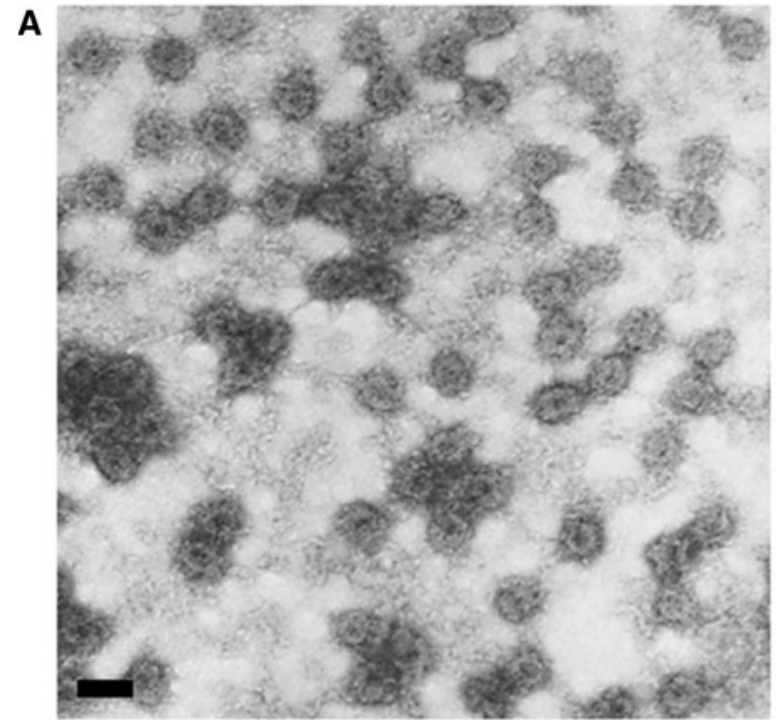

Fig. 5 Studies of the nuclear lamina from mammalian somatic cells. a Transmission electron micrograph of a negatively stained 'nuclear ghost' prepared from rat liver cells by extraction procedures. The remaining cellular material, the 'pore complex-lamina fraction,' shows an irregular arrangement of lamin filaments interspaced between nuclear pore complexes. Scale bar $100 \mathrm{~nm}$. Reprinted from (Dwyer and Blobel 1976), (C) Dwyer and Blobel (1976). Originally published in J. Cell Biol. 70(3): 581-591. b Scanning electron micrographs of an isolated mouse liver nucleus after membrane removal with Triton X-100. The nucleus is devoid of inner and outer nuclear membranes, revealing a view on nuclear pore complexes and the underlying nuclear lamina. However, no individual filaments can be detected. The image in the lower panel shows a higher
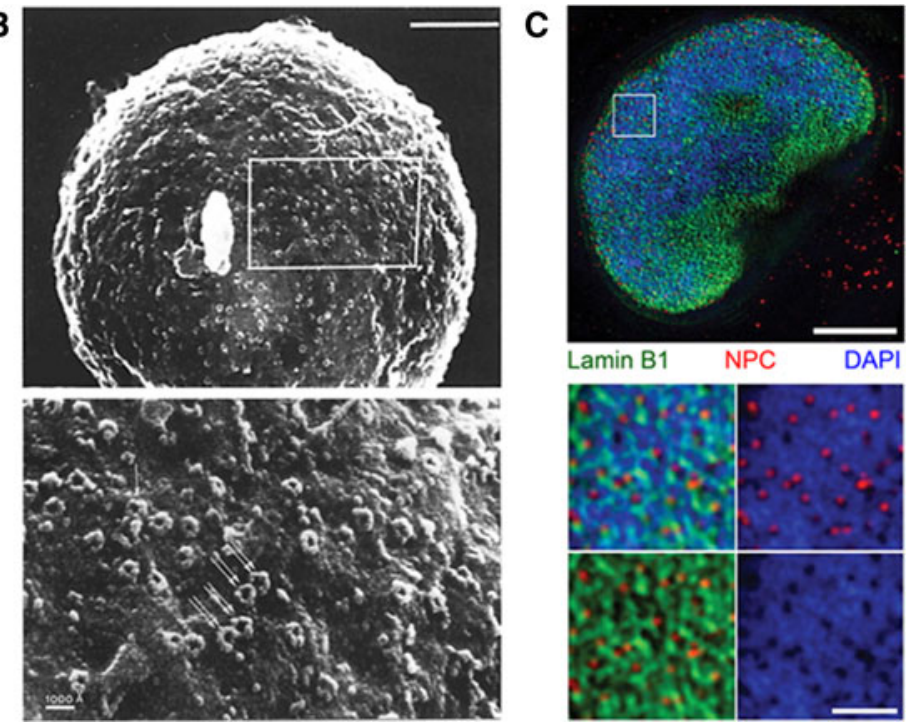

magnification of the inset in the top image with arrows highlighting nuclear pore complexes. Scale bars top $1 \mu \mathrm{m}$, bottom $100 \mathrm{~nm}$. Reprinted from (Kirschner et al. 1977), (C) Kirschner et al. (1977). Originally published in J. Cell Biol. 72(1):118-132. c Micrographs obtained from lamin B using 3D structured illumination microscopy (3D-SIM). C2C12 myoblast cells were co-immunostained with antibodies against lamin B (green) and components of the nuclear pore complex (red), and chromatin was stained with DAPI. A section of the nuclear surface was imaged (top). The white square indicates a region shown in higher magnification in the lower panel. Scale bars $5 \mu \mathrm{m}$ and $1 \mu \mathrm{m}$, respectively. Reprinted from (Schermelleh et al. 2008), with permission from AAAS 
employ microscopy techniques that support a higher resolution. Recently developed super-resolution microscopy methods such as stimulated emission depletion (STED), photoactivated localization (PALM), stochastic optical reconstruction (STORM) microscopy or structures illumination microscopy (SIM) might offer new possibilities in resolving finer details of the lamina network (Bates et al. 2007; Betzig et al. 2006; Donnert et al. 2006; Rust et al. 2006). The potential of super-resolution microscopy was demonstrated by the application of 3D-SIM to image nuclei stained with antibodies against lamin B. Micrographs of the apical side of the nucleus revealed an irregular lamin B network, interrupted by holes, in some of which nuclear pore complexes were embedded (Fig. 5c). 3D-SIM resulted in a twofold enhanced resolution compared to conventional CLSM with a resolution near $100 \mathrm{~nm}$, which corresponds to approximately twice the length of a lamin central rod domain (Schermelleh et al. 2008; Heitlinger et al. 1992).

However, to resolve individual filaments within the lamina network, in vivo, a much higher resolution is required, and therefore, electron microscopic approaches will be inevitable for deeper insights into its molecular organization. For TEM, samples must be relatively thin for electrons to pass through the specimen. The classical approach here is to prepare ultrathin sections of epoxyresin-embedded cells that were previously fixed and heavy metal stained (Kreplak et al. 2008).

An exciting application for electron microscopy, based on thinning of a vitrified cell using gallium ions, enables the sectioning of cells in a nearly unperturbed state, since neither chemical fixation nor heavy metal staining is required. Plunge frozen cells subjected to focused ion beam (FIB)-SEM are milled down by the beam, leaving only a thin vitrified lamella of cellular material. The sample can then be analyzed by cryo-electron microscopy or tomography (Rigort et al. 2012). Lamellae containing a section along the nuclear envelope surface would presumably enable tomographic reconstructions with unprecedented resolution of lamins and lamina networks. Although the application of FIB technology to biological samples is just emerging, it appears as a promising venue for the ultrastructural characterization of the lamina in living cells.

\section{Conclusions and outlook}

Structural analysis of lamin assemblies is instrumental in order to acquire a better understanding of laminopathic diseases and the variety of functions. Various techniques aimed on determining the $3 \mathrm{D}$ organization of lamin subunits, as well as lamin filaments and highly organized networks, demonstrated their potential in providing structural information on lamin assemblies. Given the organization of the lamina embedded in a compartment densely packed with lipids, proteins and chromatin, the investigation of the lamin network in vivo still remains a challenging task. New imaging techniques, such as super-resolution light microscopy or the applications of FIB to cryo-ET of vitrified cells, in combination with established methods will likely enable imaging of this protein structure at unprecedented resolution.

Interpretation of high-resolution maps of the mammalian nuclear lamina would only be possible if single filaments could be identified within the lamina context. An integrative approach for studying the nuclear lamina may be instrumental, thus combining basic knowledge obtained from in vitro studies and from the expression of single lamin networks in Xenopus oocytes with highresolution 3D-maps from the nuclear lamina of mammalian cells. The Xenopus oocyte expression system allows expressing a single or several lamins in a eukaryotic system and therefore provides a unique possibility to view the effect of specific lamins and laminbinding protein on the lamin organization. The application of cryo-ET for these studies is likely to produce pseudo-atomic maps on lamin assemblies under closeto-physiological conditions.

Extending our knowledge on the lamin organization in living cells will ultimately help to understand how mutations in these proteins alter the lamin network and network interactions, thereby causing all the various diseases that are mechanistically still far from being understood.

Acknowledgments We thank Reimer Stick for generously providing us with image material. The work is supported by a Swiss National Science Foundation grant (SNSF 31003A_141083/1) to O.M. and a postdoctoral fellowship from the German Academic Exchange Service (DAAD [D/11/44980]) to M.Z.

\section{References}

Aebi U, Cohn J, Buhle L, Gerace L (1986) The nuclear lamina is a meshwork of intermediate-type filaments. Nature 323(6088): 560-564. doi: $10.1038 / 323560 \mathrm{a} 0$

Bates M, Huang B, Dempsey GT, Zhuang XW (2007) Multicolor superresolution imaging with photo-switchable fluorescent probes. Science 317(5845):1749-1753. doi:10.1126/science.1146598

Beck LA, Hosick TJ, Sinensky M (1990) Isoprenylation is required for the processing of the lamin A precursor. J Cell Biol 110(5): 1489-1499

Ben-Harush K, Wiesel N, Frenkiel-Krispin D, Moeller D, Soreq E, Aebi U, Herrmann H, Gruenbaum Y, Medalia O (2009) The supramolecular organization of the $C$. elegans nuclear lamin filament. J Mol Biol 386(5):1392-1402. doi:10.1016/j.jmb.2008. 12.024

Betzig E, Patterson GH, Sougrat R, Lindwasser OW, Olenych S, Bonifacino JS, Davidson MW, Lippincott-Schwartz J, Hess HF (2006) Imaging intracellular fluorescent proteins at nanometer resolution. Science 313(5793):1642-1645. doi:10.1126/science. 1127344 
Dechat T, Adam SA, Taimen P, Shimi T, Goldman RD (2010a) Nuclear lamins. Cold Spring Harb Perspect Biol 2(11):a000547. doi:10.1101/cshperspect.a000547

Dechat T, Gesson K, Foisner R (2010b) Lamina-independent lamins in the nuclear interior serve important functions. Cold Spring Harb Symp Quant Biol 75:533-543. doi:10.1101/sqb.2010. 75.018

Dhe-Paganon S, Werner ED, Chi YI, Shoelson SE (2002) Structure of the globular tail of nuclear lamin. J Biol Chem 277(20): 17381-17384. doi:10.1074/jbc.C200038200

Donnert G, Keller J, Medda R, Andrei MA, Rizzoli SO, Luhrmann R, Jahn R, Eggeling C, Hell SW (2006) Macromolecular-scale resolution in biological fluorescence microscopy. Proc Natl Acad Sci USA 103(31):11440-11445. doi:10.1073/pnas.0604965103

Dubochet J, Adrian M, Chang JJ, Homo JC, Lepault J, McDowall AW, Schultz P (1988) Cryo-electron microscopy of vitrified specimens. Q Rev Biophys 21(2):129-228

Dwyer N, Blobel G (1976) A modified procedure for the isolation of a pore complex-lamina fraction from rat liver nuclei. J Cell Biol 70(3):581-591

Eckersley-Maslin MA, Bergmann JH, Lazar Z, Spector DL (2013) Lamin $\mathrm{A} / \mathrm{C}$ is expressed in pluripotent mouse embryonic stem cells. Nucleus 4(1):53-60. doi:10.4161/nucl.23384

Fridman K, Mader A, Zwerger M, Elia N, Medalia O (2012) Advances in tomography: probing the molecular architecture of cells. Nat Rev Mol Cell Biol 13(11):736-742. doi:10.1038/nrm 3453

Gall JG, Wu Z, Murphy C, Gao H (2004) Structure in the amphibian germinal vesicle. Exp Cell Res 296(1):28-34. doi:10.1016/ j.yexcr.2004.03.017

Goldberg MW, Huttenlauch I, Hutchison CJ, Stick R (2008) Filaments made from A- and B-type lamins differ in structure and organization. J Cell Sci 121(Pt 2):215-225. doi:10.1242/ jcs. 022020

Goldman AE, Maul G, Steinert PM, Yang HY, Goldman RD (1986) Keratin-like proteins that coisolate with intermediate filaments of BHK-21 cells are nuclear lamins. Proc Natl Acad Sci USA 83(11):3839-3843

Grossman E, Dahan I, Stick R, Goldberg MW, Gruenbaum Y, Medalia O (2012) Filaments assembly of ectopically expressed Caenorhabditis elegans lamin within Xenopus oocytes. J Struct Biol 177(1):113-118. doi:10.1016/j.jsb.2011.11.002

Grubisha O, Kaminska M, Duquerroy S, Fontan E, Cordier F, Haouz A, Raynal B, Chiaravalli J, Delepierre M, Israel A, Veron M, Agou F (2010) DARPin-assisted crystallography of the CC2-LZ domain of NEMO reveals a coupling between dimerization and ubiquitin binding. J Mol Biol 395(1):89-104. doi:10.1016/j.jmb. 2009.10.018

Guelen L, Pagie L, Brasset E, Meuleman W, Faza MB, Talhout W, Eussen BH, de Klein A, Wessels L, de Laat W, van Steensel B (2008) Domain organization of human chromosomes revealed by mapping of nuclear lamina interactions. Nature 453(7197): 948-951. doi:10.1038/nature06947

Heitlinger E, Peter M, Haner M, Lustig A, Aebi U, Nigg EA (1991) Expression of chicken lamin B2 in Escherichia coli: characterization of its structure, assembly, and molecular interactions. J Cell Biol 113(3):485-495

Heitlinger E, Peter M, Lustig A, Villiger W, Nigg EA, Aebi U (1992) The role of the head and tail domain in lamin structure and assembly: analysis of bacterially expressed chicken lamin A and truncated B2 lamins. J Struct Biol 108(1):74-89

Herrmann H, Aebi U (2004) Intermediate filaments: molecular structure, assembly mechanism, and integration into functionally distinct intracellular Scaffolds. Annu Rev Biochem 73:749-789. doi:10.1146/annurev.biochem.73.011303.073823
Herrmann H, Hesse M, Reichenzeller M, Aebi U, Magin TM (2003) Functional complexity of intermediate filament cytoskeletons: from structure to assembly to gene ablation. Int Rev Cytol 223:83-175

Herrmann H, Kreplak L, Aebi U (2004) Isolation, characterization, and in vitro assembly of intermediate filaments. Methods Cell Biol 78:3-24

Herrmann H, Bar H, Kreplak L, Strelkov SV, Aebi U (2007) Intermediate filaments: from cell architecture to nanomechanics. Nat Rev Mol Cell Biol 8(7):562-573. doi:10.1038/nrm2197

Holmer L, Worman HJ (2001) Inner nuclear membrane proteins: functions and targeting. Cell Mol Life Sci 58(12-13):1741-1747

Kapinos LE, Burkhard P, Herrmann H, Aebi U, Strelkov SV (2011) Simultaneous formation of right- and left-handed anti-parallel coiled-coil interfaces by a coil2 fragment of human lamin A. J Mol Biol 408(1):135-146. doi:10.1016/j.jmb.2011.02.037

Karabinos A, Schunemann J, Meyer M, Aebi U, Weber K (2003) The single nuclear lamin of Caenorhabditis elegans forms in vitro stable intermediate filaments and paracrystals with a reduced axial periodicity. J Mol Biol 325(2):241-247

Kaufmann A, Heinemann F, Radmacher M, Stick R (2011) Amphibian oocyte nuclei expressing lamin A with the progeria mutation E145K exhibit an increased elastic modulus. Nucleus 2(4):310-319. doi:10.4161/nucl.2.4.16119

Kirschner RH, Rusli M, Martin TE (1977) Characterization of the nuclear envelope, pore complexes, and dense lamina of mouse liver nuclei by high resolution scanning electron microscopy. J Cell Biol 72(1):118-132

Kitten GT, Nigg EA (1991) The CaaX motif is required for isoprenylation, carboxyl methylation, and nuclear membrane association of lamin B2. J Cell Biol 113(1):13-23

Korfali N, Wilkie GS, Swanson SK, Srsen V, de Las Heras J, Batrakou DG, Malik P, Zuleger N, Kerr AR, Florens L, Schirmer EC (2012) The nuclear envelope proteome differs notably between tissues. Nucleus 3(6):552-564. doi:10.4161/nucl.22257

Kreplak L, Richter K, Aebi U, Herrmann H (2008) Electron microscopy of intermediate filaments: teaming up with atomic force and confocal laser scanning microscopy. Methods Cell Biol 88:273-297. doi:10.1016/S0091-679x(08)00415-9

Kubben N, Voncken JW, Demmers J, Calis C, van Almen G, Pinto Y, Misteli T (2010) Identification of differential protein interactors of lamin A and progerin. Nucleus 1(6):513-525. doi:10.4161/ nucl.1.6.13512

Lin F, Worman HJ (1993) Structural organization of the human gene encoding nuclear lamin A and nuclear lamin C. J Biol Chem 268(22):16321-16326

Lloyd DJ, Trembath RC, Shackleton S (2002) A novel interaction between lamin A and SREBP1: implications for partial lipodystrophy and other laminopathies. Hum Mol Genet 11(7): 769-777

Loewinger L, McKeon F (1988) Mutations in the nuclear lamin proteins resulting in their aberrant assembly in the cytoplasm. EMBO J 7(8):2301-2309

Luderus ME, de Graaf A, Mattia E, den Blaauwen JL, Grande MA, de Jong L, van Driel R (1992) Binding of matrix attachment regions to lamin B1. Cell 70(6):949-959

Mattout A, Goldberg M, Tzur Y, Margalit A, Gruenbaum Y (2007) Specific and conserved sequences in D. melanogaster and C. elegans lamins and histone $\mathrm{H} 2 \mathrm{~A}$ mediate the attachment of lamins to chromosomes. J Cell Sci 120 (Pt 1):77-85. doi: $10.1242 /$ jcs. 03325

McKeon FD, Kirschner MW, Caput D (1986) Homologies in both primary and secondary structure between nuclear envelope and intermediate filament proteins. Nature 319(6053):463-468. doi: $10.1038 / 319463 \mathrm{a} 0$ 
Mendez-Lopez I, Worman HJ (2012) Inner nuclear membrane proteins: impact on human disease. Chromosoma 121(2): 153-167. doi:10.1007/s00412-012-0360-2

Moir RD, Quinlan RA, Stewart M (1990) Expression and characterization of human lamin C. FEBS Lett 268(1):301-305

Parry DA (2005) Microdissection of the sequence and structure of intermediate filament chains. Adv Protein Chem 70:113-142. doi:10.1016/S0065-3233(05)70005-X

Peter M, Kitten GT, Lehner CF, Vorburger K, Bailer SM, Maridor G, Nigg EA (1989) Cloning and sequencing of cDNA clones encoding chicken lamins $\mathrm{A}$ and $\mathrm{B} 1$ and comparison of the primary structures of vertebrate A- and B-type lamins. J Mol Biol 208(3):393-404

Qin Z, Kalinowski A, Dahl KN, Buehler MJ (2011) Structure and stability of the lamin A tail domain and HGPS mutant. J Struct Biol 175(3):425-433. doi:10.1016/j.jsb.2011.05.015

Rankin J, Ellard S (2006) The laminopathies: a clinical review. Clin Genet 70(4):261-274. doi:10.1111/j.1399-0004.2006.00677.x

Rigort A, Bauerlein FJB, Villa E, Eibauer M, Laugks T, Baumeister W, Plitzko JM (2012) Focused ion beam micromachining of eukaryotic cells for cryoelectron tomography. Proc Natl Acad Sci USA 109(12):4449-4454. doi:10.1073/pnas.1201333109

Ruan J, Xu C, Bian C, Lam R, Wang JP, Kania J, Min J, Zang J (2012) Crystal structures of the coil $2 B$ fragment and the globular tail domain of human lamin B1. FEBS Lett 586(4): 314-318. doi:10.1016/j.febslet.2012.01.007

Rust MJ, Bates M, Zhuang XW (2006) Sub-diffraction-limit imaging by stochastic optical reconstruction microscopy (STORM). Nat Methods 3(10):793-795. doi:10.1038/Nmeth929

Schermelleh L, Carlton PM, Haase S, Shao L, Winoto L, Kner P, Burke B, Cardoso MC, Agard DA, Gustafsson MGL, Leonhardt H, Sedat JW (2008) Subdiffraction multicolor imaging of the nuclear periphery with 3D structured illumination microscopy. Science 320(5881):1332-1336. doi:10.1126/science.1156947

Sennhauser G, Grutter MG (2008) Chaperone-assisted crystallography with DARPins. Structure 16(10):1443-1453. doi:10.1016/ j.str.2008.08.010

Shimi T, Pfleghaar K, Kojima S, Pack CG, Solovei I, Goldman AE, Adam SA, Shumaker DK, Kinjo M, Cremer T, Goldman RD (2008) The A- and B-type nuclear lamin networks: microdomains involved in chromatin organization and transcription. Genes Dev 22(24):3409-3421. doi:10.1101/gad.1735208

Shumaker DK, Lopez-Soler RI, Adam SA, Herrmann H, Moir RD, Spann TP, Goldman RD (2005) Functions and dysfunctions of the nuclear lamin Ig-fold domain in nuclear assembly, growth, and Emery-Dreifuss muscular dystrophy. Proc Natl Acad Sci USA 102(43):15494-15499. doi:10.1073/pnas.0507612102
Simon DN, Zastrow MS, Wilson KL (2010) Direct actin binding to Aand B-type lamin tails and actin filament bundling by the lamin A tail. Nucleus 1(3):264-272. doi:10.4161/nucl.1.3.11799

Sinensky M, Fantle K, Trujillo M, McLain T, Kupfer A, Dalton M (1994) The processing pathway of prelamin A. J Cell Sci 107(Pt 1):61-67

Starger JM, Brown WE, Goldman AE, Goldman RD (1978) Biochemical and immunological analysis of rapidly purified 10-nm filaments from baby hamster kidney (BHK-21) cells. J Cell Biol 78(1):93-109

Steinert P, Zackroff R, Aynardi-Whitman M, Goldman RD (1982) Isolation and characterization of intermediate filaments. Methods Cell Biol 24:399-419

Stick R, Goldberg MW (2010) Oocytes as an experimental system to analyze the ultrastructure of endogenous and ectopically expressed nuclear envelope components by field-emission scanning electron microscopy. Methods 51(1):170-176. doi:10.1016/ j.ymeth.2010.01.015

Strelkov SV, Burkhard P (2002) Analysis of alpha-helical coiled coils with the program TWISTER reveals a structural mechanism for stutter compensation. J Struct Biol 137(1-2):54-64. doi: 10.1006/jsbi.2002.4454

Strelkov SV, Schumacher J, Burkhard P, Aebi U, Herrmann H (2004) Crystal structure of the human lamin A coil 2B dimer: implications for the head-to-tail association of nuclear lamins. J Mol Biol 343(4):1067-1080. doi:10.1016/j.jmb.2004.08.093

Taimen P, Pfleghaar K, Shimi T, Moller D, Ben-Harush K, Erdos MR, Adam SA, Herrmann H, Medalia O, Collins FS, Goldman AE, Goldman RD (2009) A progeria mutation reveals functions for lamin A in nuclear assembly, architecture, and chromosome organization. Proc Natl Acad Sci USA 106(49):20788-20793. doi:10.1073/pnas.0911895106

Vorburger K, Lehner CF, Kitten GT, Eppenberger HM, Nigg EA (1989) A second higher vertebrate B-type lamin: cDNA sequence determination and in vitro processing of chicken lamin B2. J Mol Biol 208(3):405-415

Worman HJ (2012) Nuclear lamins and laminopathies. J Pathol 226(2):316-325. doi:10.1002/path.2999

Worman HJ, Lazaridis I, Georgatos SD (1988) Nuclear lamina heterogeneity in mammalian cells. Differential expression of the major lamins and variations in lamin B phosphorylation. J Biol Chem 263(24):12135-12141

Yahav T, Maimon T, Grossman E, Dahan I, Medalia O (2011) Cryoelectron tomography: gaining insight into cellular processes by structural approaches. Curr Opin Struct Biol 21(5):670-677. doi: 10.1016/j.sbi.2011.07.004 